\title{
Higher orders of the high-temperature expansion for the Ising model in three dimensions
}

\author{
H. Arisue ${ }^{a}$, T. Fujiwara ${ }^{\mathrm{b}} \dagger$ and K. Tabata ${ }^{a \ddagger}$ \\ ${ }^{a}$ Osaka Prefectural College of Technology, Saiwai-cho 26-12, Neyagawa, Osaka 572-8572, Japan \\ ${ }^{\text {b} F a c u l t y ~ o f ~ G e n e r a l ~ S t u d i e s, ~ K i t a s a t o ~ U n i v e r s i t y, ~ K i t a s a t o ~ 1-15-1, ~ S a g a m i h a r a, ~ K a n a g a w a ~ 228-8555, ~}$ \\ Japan
}

The new algorithm of the finite lattice method is applied to generate the high-temperature expansion series of the simple cubic Ising model to $\beta^{50}$ for the free energy, to $\beta^{32}$ for the magnetic susceptibility and to $\beta^{29}$ for the second moment correlation length. The series are analyzed to give the precise value of the critical point and the critical exponents of the model.

\section{INTRODUCTION}

The finite lattice method 123 is a powerful tool to generate the exact high- and lowtemperature series and other exact expansion series for the spin models in the infinite volume limit. Using this method we can skip the job of listing up all the relevant diagrams and of counting the number they appear, which is inevitable in the graphical method. Then the main task is reduced to the calculation of the partition function for the appropriate finite size lattices, which can be done efficiently by the site-by-site integration using the transfer matrix formalism [45] without the graphical technique. The CPU time and the computer memory needed to obtain the series to order $N$ increase exponentially with $N$ in two dimensions, while they grow up exponentially with $N^{2}$ in three dimensions. Thus the finite lattice method has been extremely effective in two dimensions, but it has generated the series only in some limited cases in three dimensions.

Recently we presented the new algorithm of the finite lattice method 67] in which the CPU time and the computer memory increase exponentially with $N \log N$. It enabled us to extend the high-temperature expansion series for the free

\footnotetext{
*email address:arisue@las.osaka-pct.ac.jp

$\dagger$ email address:fujiwara@clas.kitasato-u.ac.jp

¥email address:tabata@las.osaka-pct.ac.jp
}

energy of the simple cubic Ising model to order $\beta^{46}$ from the previous series of order $\beta^{26}$, which was given by the previous algorithm of the finite lattice method, and to order $\beta^{32}$ for the magnetic susceptibility from the previous series of order $\beta^{25}$, which was given by the graphical method.

Here we apply the method newly to the hightemperature expansion of the second moment correlation length to order $\beta^{29}$ and also extend the series for the free energy to order $\beta^{50}$. The series themselves will be presented elsewhere 8 and we give here the result of the analysis of the newly generated series combined with the series for the magnetic susceptibility already obtained.

\section{SUSCEPTIBILITY AND CORRELA- TION LENGTH}

The magnetic susceptibility $\chi$ and the second moment correlation length $\xi_{2}$ are expected to behave like

$$
\begin{gathered}
\chi=A_{1}\left(\beta_{c}-\beta\right)^{-\gamma}\left[1+c_{1}\left(\beta_{c}-\beta\right)^{\Delta}+\cdots\right] \\
\xi_{2}{ }^{2}=A_{2}\left(\beta_{c}-\beta\right)^{-2 \nu}\left[1+c_{2}\left(\beta_{c}-\beta\right)^{\Delta}+\cdots\right]
\end{gathered}
$$

Here $\beta_{c}$ is the critical point and $\Delta$ is the leading correction-to-scaling exponent.

There are several evaluation of the value of $\Delta$ to be around 0.5. To make more precise evaluation we plot $2 \nu$ versus $\Delta$ in Figure 1 in the analysis of 


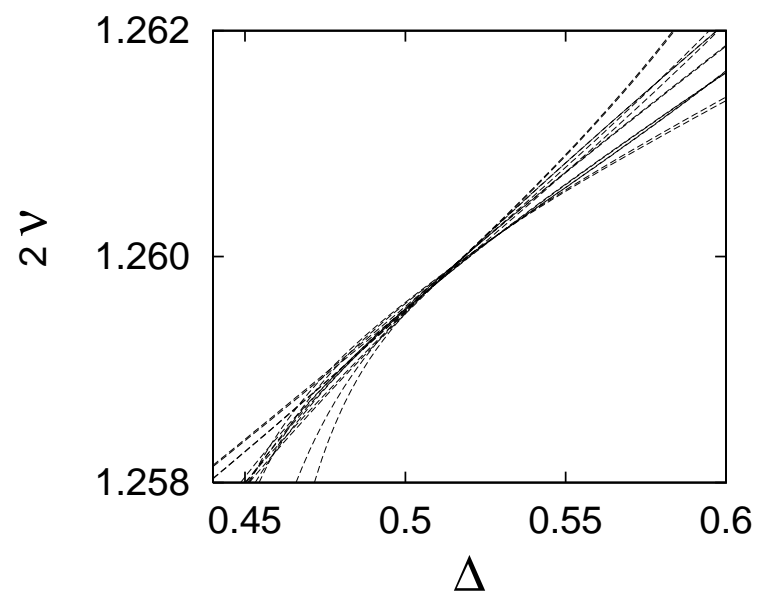

Figure 1. Critical exponent $2 \nu$ plotted versus $\Delta$ in the M2 method of the series analysis for $\xi_{2}{ }^{2}$.

the $\xi_{2}$ series by the M2 method 9. The narrow crossing region of the lines gives $\Delta=0.51(1)$.

The modified ratio series 10 .

$$
\left(\beta_{c}\right)_{n} \equiv\left(\frac{a_{n-2} a_{n-3}}{a_{n} a_{n-1}}\right) \exp \left[\frac{\left(s_{n}+s_{n-2}\right)}{2 s_{n}\left(s_{n}-s_{n-2}\right)}\right]
$$

with

$s_{n}=\left(\ln \left(\frac{a_{n-2}^{2}}{a_{n} a_{n-4}}\right)^{-1}+\ln \left(\frac{a_{n-3}^{2}}{a_{n-1} a_{n-5}}\right)^{-1}\right) / 2$

made of the expantion coefficients $a_{n}$ for each quantity is expected to behave like

$\left(\beta_{c}\right)_{n}=\beta_{c}+\frac{b}{n^{1+\Delta}}+\cdots$.

Although the modefied ratio series for the longest series of each of the $\chi$ and $\xi_{2}{ }^{2}$ can be fitted well by the first two terms in Eq. (2) with $\Delta=0.51$, there is still a considerably large ambiguity in extrapolating the plot to $n \rightarrow \infty$.

Then we take the following combined quantity of the susceptibility and the correlation length.

$\chi /\left(\xi^{2}\right)^{\omega}$

$=A_{3}\left(\beta_{c}-\beta\right)^{-\gamma+2 \omega \nu}\left[1+c_{3}\left(\beta_{c}-\beta\right)^{\Delta}+\cdots\right]$

We can expect that, if we take an appropriate value of $\omega$, the third and later terms are very

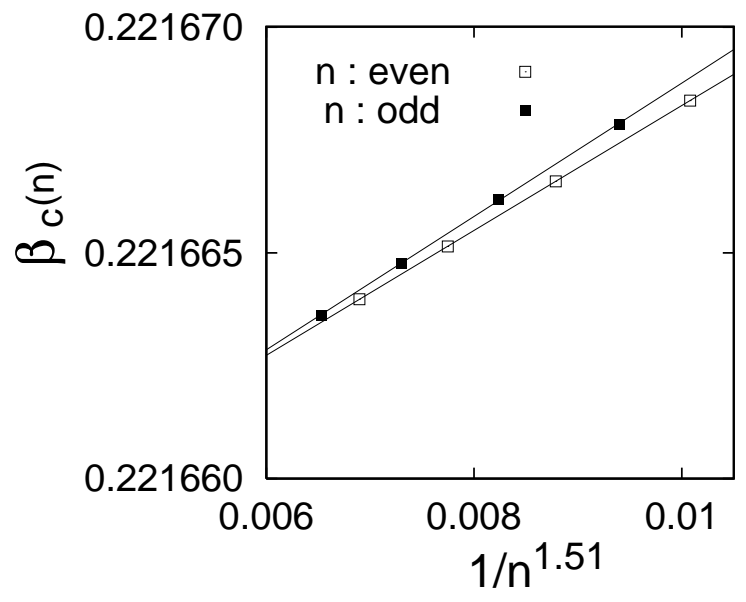

Figure 2. Modified ratio series for the combination of $\chi$ and $\xi_{2}$ with $\omega=0.055$ plotted versus $1 / n^{1+\Delta}$

small and the ratio series (11) for this quantity can be fitted quite well by the first two terms in Eq. (2). In fact we find as in Figure 2 that the situation is realized for $\omega=0.055$ for even series of $n=22-28$ giving $\beta_{c}=0.2216545(1)$ if we assume $\Delta=0.51(1)$. Similarly the combined quantity $\xi_{2}{ }^{2} / \chi^{\omega^{\prime}}$ with $\omega^{\prime}=-0.15$ gives $\beta_{c}=0.2216542(4)$ with the same value of $\Delta$ assumed.

There are two estimates for the critical point of the model by the recent large scale numerical simulations. One is $\beta_{c}=0.22165459(10)$ by Blöte et al. 11] using the cluster algorithm and the other is $\beta_{c}=0.2216545(15)$ 12 by Ito et al. using the non-equilibrium relaxation method. They are contradictory to each other. Our estimation by the analysis of the high-temperature series is consistent with these estimates.

As for the critical exponents the modified ratio series for the combination (3) of the magnetic susceptibility and the second moment correlation length gives $\gamma=1.2369(3)$ and $\nu=0.6298(3)$ for $\Delta=0.51(1)$. These values are consistent with the estimate of $\gamma=1.2373(2)$ and $\nu=0.6301(2)$ by the high-temperature expansion of the generalized Ising model 13 and of $\gamma=1.2396(13)$ and $\nu=0.6304(13)$ by the perturbative renormalization method 14]. 


\section{SPECIFIC HEAT}

For the critical exponent of the specific heat

$$
\begin{aligned}
C_{H} & \equiv \beta^{2} \frac{d^{2} f}{d \beta^{2}} \\
& =A_{4}\left(\beta_{c}-\beta\right)^{-\alpha}\left[1+c_{4}\left(\beta_{c}-\beta\right)^{\Delta}+\cdots\right],
\end{aligned}
$$

the ratio series

$$
\begin{aligned}
\alpha_{n} & \equiv\left[\beta_{c}^{2} \frac{a_{2 n}}{a_{2 n-2}}-1\right] n+1 \\
& =\alpha+\frac{b}{n^{\Delta}}+\frac{c}{n}+\cdots
\end{aligned}
$$

with $n \leq 50$ gives $\alpha=0.1035 \pm 0.0005$ for $\beta_{c}=0.2216546(1)$ and $\Delta=0.51(1)$. This estimate of $\alpha$ is consistent with that by the inhomogeneous differential approximation for the hightemperature series. From the hyperscaling relation $\alpha=2-d \nu$, the range of $\nu=0.6295-0.6305$, within which all of the recent precise estimates of the critical exponent for the correlation length falls, gives $\alpha=0.1085-0.1115$. Thus there is a discrepancy between the estimate of $\alpha$ from the high-temperature series of the specific heat and the estimate from the hyperscaling relation.

\section{SUMMARY AND DISCUSSION}

We have calculated the high-temperature series for the second moment correlation length of the simple cubic Ising model using the new algorithm of the finite lattice method. Combining this series with the previously obtained series for the magnetic susceptibility, we have given precise estimates of the critical point and the critical exponents, which is consistent with other recent estimates. We have also extended the series for the free energy, which gives the estimate of the critical exponent for the specific heat, which is inconsistent with that derived from the critical exponent of the correlation length using the hyperscaling relation.

The new algorithm can also be applied to the high-temperature expansion of the models that have continuous spin variables such as the XY model in three dimensions. Especially it is important to calculate the high order series of the high-temperature expansion for the XY model, since the phase transition of this model belongs to the same universality class as the lambda transition of the helium and the specific heat exponent at this phase transition point was observed very precisely in the Space Shuttle experiment 15.

Furthermore the basic idea of the new algorithm can be used in the low-temperature expansion for the spin models. We can expect that the new algorithm will enable us to generate the series for these models that are much longer than the presently available series.

\section{REFERENCES}

1. T. de Neef and I. G. Enting, J. Phys. A 10, 801 (1977); I. G. Enting, J. Phys. A 11, 563 (1978); Nucl. Phys. B (Proc. Suppl.) 47, 180 (1996).

2. H. Arisue and T. Fujiwara, Prog. Theor. Phys. 72, 1176 (1984); H. Arisue, Nucl. Phys. B (Proc. Suppl.) 34, 240 (1994).

3. M. Creutz, Phys. Rev. B 43, 10659 (1991).

4. I. G. Enting, J. Phys. A 13, 3713 (1980).

5. G. Bhanot, J. Stat. Phys. 60, 55 (1990).

6. H. Arisue and T. Fujiwara, Phys. Rev. E 67, 066109 (2003).

7. H. Arisue and T. Fujiwara, Phys. B (Proc. Suppl.) 119 (2003).

8. H. Arisue, T. Fujiwara and K. Tabata, in preparation.

9. J. Adler, M. Moshe and V. Privman, Phys. Rev. B 261411 (1983).

10. J. Zinn-Justin, J. Physique(France) 42, 783 (1981)

11. H. W. J. Blöte, L. N. Shchur and A. L. Talapov, Int. Journ. Mod. Phys. C 10, 137 (1999).

12. N. Ito, S. Fukushima, H. Watanabe and Y. Ozeki, in "Computer Simulation Studies in Condensed Matter Physics XIV" ed. D. P. Landau, S. P. Lewis and H. B. Schuettler (Sprinter-Verlag, 2002) p.27.

13. M. Campostrini, P. Rossi and E. Vicari, A. Pelissetto, Phys. Rev. E 65066127 (2002).

14. R. Guida and J. Zinn-Justin, Nucl. Phys. B 489, 626 (1997); J. Phys. A 31, 8103 (1998).

15. J. A. Lipa, D. R. Swanson and J. A. NIssen, Phys. Prev. Lett. 76, 944 (1996). 